Rev. salud pública. 14 sup (1): 18-31, 2012

\title{
Neoliberalismo en salud: La tortura de trabajadoras y trabajadores del Instituto Materno Infantil de Bogotá
}

\section{Neoliberalism in health: the torture of the health care workers of the Bogota's Instituto Materno Infantil (child and maternity hospital)}

\author{
César Abadía B., María Y. Pinilla A., Katerine Ariza R. y Héctor C. Ruíz S. \\ Centro de Estudios Sociales. Facultad de Ciencias Humanas. Universidad Nacional de Colombia. \\ Bogotá.ceabadiab@unal.edu.co,cesar_abadia@post.harvard.edu \\ Recibido 21 julio 2009/Enviado para Modificación 5 Mayo 2010/Aceptado 12 Mayo 2009
}

\section{RESUMEN}

Objetivos Relacionar históricamente las transformaciones más significativas del Instituto Materno Infantil (IMI) en su proceso de crisis, cierre y liquidación con las experiencias de sus trabajadores/as. Encontrar elementos vivenciales y teóricos que interconecten el proceso de privatización de la salud con las experiencias de resistencia y dolor/sufrimiento de trabajadores/as.

Métodos Etnografía inscrita en corrientes críticas y apoyada en trabajo de campo constante y colectivo, investigación histórica (fuentes primarias y secundarias) y entrevistas semiestructuradas con cinco mujeres que trabajaron por más de quince años en el IMI.

Resultados Una línea del tiempo con cuatro periodos principales: Los años de gloria (hasta 1990), Llega el neoliberalismo (1990-2000), La crisis y las resistencias (2001-2005) y Liquidación (2006-). La narrativa de las mujeres entrevistadas devela múltiples agresiones que se intensificaron desde el 2006 generando dolor/ sufrimiento, relatos que ilustran violaciones a sus derechos humanos y laborales. Discusión Proponemos analizar las conexiones entre los diferentes tipos de violencia y el dolor/sufrimiento bajo la categoría tortura, entendida como acciones violentas que causan dolor físico-emocional, las cuales son ejecutadas por actores de poder sobre otros que desafían alterarlo. Enfatizamos en las burocracias, el confinamiento, los agentes torturadores y los resquebrajamientos a la unidad mente/cuerpo para argumentar que esta relación neoliberalismo y tortura pretende eliminar los últimos trabajadores/as de la salud del país con garantías laborales para avanzar en la acumulación de capital que genera la creciente sobreexplotación del trabajo y la mercantilización de la salud.

Palabras Clave: Capitalismo, trabajo, tortura, violencia, dolor, sufrimiento, Colombia. (fuente: DeCS, BIREME). 


\section{ABSTRACT}

Objectives To link, from a historical point of view, the most significant transformations of the Instituto Materno Infantil (IMI) [the oldest child and maternity hospital of the country] during its process of crisis, closure and liquidation with the experiences of the hospital workers. To find experience-based and theoretical elements that can interconnect the process of health care privatization of the country with the workers' experiences of resistance and pain/suffering.

Methods Critically-oriented ethnography based on continuous collective field work, historical research (primary and secondary sources) and semi-structured interviews with 5 women who worked at the IMI for more than 15 years.

Results: A time line of 4 main periods: Los años de gloria [The golden years] (up to 1990); Llega el neoliberalismo [Neoliberalism arrives] (1990-2000); La crisis y las resistencias [Crisis and resistances] (2001-2005); and Liquidación [Liquidation (2006-20??)]. The narratives of the interviewed women unveil multiple aggressions that have intensified since 2006, have caused pain and suffering and are examples of violations of human and labour rights.

Discussion We suggest to analyze the links between the different kinds of violence and pain and suffering as torture. This category is defined as the set of violent actions that cause physical and emotional pain, which are performed by actors in positions of power over other people who challenge that power and are part of modern States' ideological principles around a defined moral social order. For the IMI workers' case, the ideological principle that is being challenged is health care neoliberalism. From the analyses of bureaucracy, confinement, torturing agents, and the breaking-off of the body-mind unit we conclude that this relationship between neoliberalism and torture aims to eliminate the last health care workers of the country who had job stability and full-benefits through public labour contracts. Their elimination furthers the accumulation of capital generated by increasing overexploitation of labour and commodification of health care.

Key Words: Capitalism, employment, torture, violence, pain, suffering, Colombia (source: Mesh,NLM)

$\mathrm{E}$ trabajo en el neoliberalismo. Históricamente, las distintas actividades consideradas como trabajo han sido pensadas como cosustanciales al orden social y a la esencia misma del ser humano. A las perspectivas que ven al trabajo como necesario, humano y emancipador se suman aquellas que ven en esta actividad formas de subordinación, degradación, alienación o esclavismo, convirtiendo la cuestión del trabajo en una dimensión dual y dialéctica (1). Reflexiones académicas sobre la relación entre los desarrollos del capitalismo y las dinámicas sociales alrededor del trabajo son las más pertinentes para entender el mundo del trabajo contemporáneo, en donde pasamos del modelo fordista-taylorista del keynesianismo de los Estados de Bienestar de Europa Occidental, al 
modelo de acumulación flexible del neoliberalismo, caracterizado por desempleo estructural, deslocalización y transnacionalización de formas de súper explotación, precarización de las condiciones laborales, expansión de asalariados en labores de servicios, exclusión de jóvenes y viejos, usufructo de las virtudes de los trabajadores y nuevas formas de división sexual del trabajo (2-4).

En América Latina, el Estado de Bienestar nunca se consolidaría (5). Durante los setentas y ochentas, la crisis del petróleo y el endeudamiento progresivo llevarían a la mayoría de los países de América Latina y el Caribe a un profundo desajuste social y económico. Las agencias financieras multinacionales señalaron a los Estados como los responsables debido a su supuesta ineficiencia para controlar el déficit público, principalmente el aumento en los gastos sociales en seguridad social (6-10). La revitalización de los postulados liberales (libre mercado, libertad individual y no injerencia del Estado) llevó a proponer reformas profundas en los sistemas de protección social conocidas como políticas de ajuste estructural, que en América Latina adoptarían un modelo conocido como neoasistencialismo, caracterizado por una combinación entre aseguramiento privado y asistencia pública focalizada (5). Estas reformas implicarían transformaciones en el mundo del trabajo y en la seguridad social llevando a la eliminación de los contratos laborales y al dominio de los mercados de aseguramiento en salud y pensiones por parte de empresas transnacionales $(7,8,10,11)$.

\section{Neoliberalismo y trabajo en Colombia}

La Ley 50 de 1990 reformó el código sustantivo del trabajo vigente desde 1950. Argumentando que los costos y la rigidez del mercado eran la razón fundamental de los desajustes laborales (12), se introdujeron entonces los contratos a término fijo, el salario integral y la pérdida de la retroactividad de las cesantías. Con la premisa de crear millones de empleos, el congreso aprueba la Ley 789 de 2002, que amplía la jornada laboral hasta las diez de la noche y disminuye el pago por horas extras, trabajo nocturno, dominical o festivo. Sin embargo una investigación concluye que esta reforma aumentó el sub-empleo, mientras que las tasas de empleo y los ingresos de los trabajadores disminuyeron (12).

Basada en este estudio, la Central Unitaria de Trabajadores (CUT) demandó dicha reforma laboral. La Procuraduría General de la Nación 
emitió un concepto invitando a la Corte Constitucional a declarar inexequible varios artículos de la reforma por considerarlos un retroceso en la protección del derecho al trabajo y no cumplir sus objetivos. Sin embargo la Corte ratifica el carácter constitucional de la misma.

Bajo la nueva modalidad de prestación de servicios, los trabajadores de la salud se encuentran en una situación caracterizada por inseguridad laboral, malos pagos y ausencia de prestaciones sociales, perdiendo la posibilidad de pensar en planes futuros (13). Tecnologías de control, propias de las empresas privadas de servicios, introducen supuestos valores como autonomía, responsabilidad, compromiso, flexibilidad, competencia y emprendimiento para que los mismos trabajadores auto-gestionen su explotación (14). Adicionalmente, la reforma a la seguridad social conocida como Ley 100 de 1993, con la lógica de empresarización de las instituciones de salud $(14,15)$, llevó a la pérdida de autonomía profesional y perjudicó la calidad de la atención (16-18).

La investigación en el Instituto Materno Infantil (IMI).

Esta investigación inició en 2005 y se propuso indagar por los impactos de las reestructuraciones legales y administrativas del sistema de salud en la crisis y viabilidad financiera del IMI reconocido como hospital insignia de la maternidad en el país. De esta primera parte, los datos arrojaron que la crisis se sentía muy fuerte en los y las trabajadoras, mas no en las pacientes (19).

En el 2006, empezamos una etnografía, aún vigente, enmarcada en la investigación-acción. Durante el 2007 y 2008 se llevaron a cabo trabajos de "organización histórica" y recuperación de la memoria institucional a través de conversaciones y talleres con trabajadores/as y docentes, que contrastamos con archivos de fuentes primarias y lecturas de fuentes secundarias.

De estos años de investigación, destacamos las dificultades económicas, emocionales y familiares que se evidenciaban como dolor/sufrimiento (20, 21) en las distintas narrativas y se relacionaban de forma muy directa con las transformaciones del IMI.

En nuestro acompañamiento a trabajadores/as del IMI, hemos hecho varios esfuerzos por entender la relación entre procesos históricos de 
ordenamiento social de la salud en Colombia y las múltiples violencias que se narran y sienten en sus voces y cuerpos. Es por esto que en el primer semestre de 2009 iniciamos una temporada de campo cuyos objetivos son: 1) Realizar una recuperación histórica que relacione las transformaciones más significativas del hospital con las experiencias de trabajadores/as. 2) Encontrar elementos vivenciales y teóricos que nos permitan interconectar los múltiples eventos y acciones violentas del proceso de privatización de la salud con las experiencias de resistencia y dolor/sufrimiento de trabajadores/as.

\section{METODOLOGIA}

Etnografía inscrita en corrientes críticas y apoyada en:

- Trabajo de campo constante y colectivo.

- Investigación histórica con fuentes primarias (materiales de archivo) y fuentes secundarias.

- Entrevistas semiestructuradas con cinco mujeres que trabajaron por más de quince años en la institución. Tres que aún esperan su liquidación y asisten regularmente al IMI: una Auxiliar de Enfermería (AE), una Técnica de Radiología (TR) y una Secretaria (S). Una Enfermera Jefe (EJ) que consiguió otro trabajo en una clínica privada. Y una Terapeuta Ocupacional (TO) que fue contratada por el Hospital La Victoria (HLV). La guía de entrevista y su abordaje metodológico se apoyó en discusiones sobre narrativas de trauma (22) y en una versión validada para Colombia de la escala diagnóstica del trastorno de estrés postraumático (23).

\section{RESULTADOS}

\section{Línea de Tiempo}

Del análisis histórico y de conversaciones informales diagramamos una línea de tiempo del Materno dividida en cuatro periodos.

- Los años de gloria (hasta 1990). En este periodo sobresale la creación y consolidación del Programa Madre Canguro y el cambio en la administración del hospital que pasa de la Beneficencia de Cundinamarca a una fundación privada.

- Llega el neoliberalismo (1990-2000). Se crean e implementan la ley 50 de 1990 y la ley 100 de 1993. 
- La crisis y las resistencias (2001-2005). Es cerrado el Hospital San Juan de Dios (HSJD), que junto al IMI conformaban la principal unidad de atención pública del país. El Instituto de Seguros Sociales (ISS) suspende el contrato de servicios y embarga al IMI por no haber cumplido con los pagos de salud y pensión acordados para las/os trabajadores del hospital. Con el fin del contrato con el ISS se reduce el número de pacientes atendidos en el IMI, hecho que dificulta, entre otras cosas, cubrir la nómina y responder a los acreedores. Como eventos legales se encuentran la Ley 735 por medio de la cual se reconoce al HSJD y al IMI como patrimonio cultural de la nación y la devolución del carácter de hospital público por parte del Consejo de Estado quien declaró nula la fundación privada.

- Liquidación (2006 en adelante). Proceso aún vigente liderado por la agente liquidadora Ana Karenina Gaula, contratada por el ex-gobernador de Cundinamarca y ex-convicto Pablo Ardila. Se destaca en 2006 el subarrendamiento del IMI al Hospital de La Victoria -el cual ingresa a los espacios del hospital acompañado del Escuadrón Móvil Antidisturbios (ESMAD)- y la posterior fragmentación del IMI en dos territorios: La Victoria sede IMI, y "La Carpa", espacio de resistencia en el cual trabajadoras/es esperan que se resuelva su situación laboral. En el 2008 la Corte Constitucional ratifica el carácter público de los hospitales y sus trabajadores/as, pero define la validez de los contratos laborales solo hasta el 2006.

2. Las trabajadoras del IMI. Narrativas desde el dolor y el Sufrimiento

Para las cinco entrevistas se utilizó la línea del tiempo como ayuda visual buscando enfocar cronológicamente los relatos y compararlos con los datos históricos recuperados en los años anteriores. A continuación presentamos una narrativa que intenta ligar las transformaciones al interior del IMI con las vivencias de las entrevistadas, incluyendo un fragmento de una entrevista realizada con otra secretaria en el 2005 que incluimos por su pertinencia para el presente artículo y aparece como primer relato de la sección La crisis y las resistencias (2001-2005).

Los años de gloria (hasta 1990)

AE: Yo me formé en la escuela Villaveces de Acosta que era del IMI, la mejor del país. Cuando volví al Materno empecé a hacer reemplazos hasta 1987 cuando me posesionaron del cargo y logré tener una estabilidad laboral. 
EJ: El trabajo era intenso y el nivel de exigencia era muy alto. Los docentes también exigían mucho para la atención. En esa época estábamos intervenidos por el Ministerio de Salud y la plata no alcanzaba. Nos descontaban salud y pensión pero ellos no pagaban, se pueden imaginar que hay personas que trabajaron 20 años y solo tenían 60 semanas cotizadas... no pagaban el sena, la luz, y luego debíamos millones.

\section{Llega el neoliberalismo (1990-2000)}

AE: Desde que entré al IMI venía escuchando que se iba a acabar. Desde que cerraron el San Juan yo tenía la visión de que esto lo iban a cerrar... si cerraron ese elefante tan grande como no van a cerrar esto que es tan pequeñito.

\section{La crisis y las resistencias (2001-2005)}

S: [La primera crisis grande fue] hace como tres años cuando ya no tenía con que matricular los niños, empecé a atrasarme en el colegio, no tenía con qué terminar de pagar ese año... Mi salario básico era de 466 mil pesos con las arandelas, que la antigüedad y todo eso, eran más o menos unos 580. Pero de esos me descontaban en ese momento, 300 de un préstamo que hicimos para una vivienda que a la final no se pudo comprar y perdimos plata y todo. De esos 280, para yo poder matricular a mi niña la más pequeña el año pasado hice un préstamo en el banco y me descontaban 147, entonces me quedaban como 90 mil, creo, porque como ahí deducen, yo recibía como 80 y de eso tenía que pagar 50 de otro préstamo que me tocó hacer, ya ni recuerdo cuando. Entonces a mí me quedaban como 30 mil pesos de sueldo y teníamos otros dos préstamos... Entonces, estamos endeudadísimos.

AE: Afortunadamente había gente que trabajaba en otros hospitales y uno les cubría el turno, y ellos le colaboraban a uno, le pagaban, eso era un aporte porque lo de acá se demoraba mucho y a veces era como incierto. Y eso no fue en el 2005, eso fue antes, nosotros llevábamos como tres años con retrasos en los sueldos, llegamos a durar ocho meses sin sueldo.

EJ: Atender un parto era exótico, cuando antes había pacientes que tocaba atender en camilla... el tema de no tener pacientes fue durísimo, cuando la gente empieza a estar sentadita, empieza a caer en una cosa como si no importara, como una apatía... estar sin pacientes no era bueno, habían más peleas, yo al final estaba como agotada no quería ni pararme.

AE: Yo siempre fui del turno de la noche, y cuando veía que en mi servicio solo había dos bebés, y en otros había uno y mamás no había ninguna, ahí ya era inminente. Un día llegué y había un bebé, y llegó la ambulancia por ese bebé y quedó Juan David, el último bebé del IMI y todo el mundo llorando. Fue muy terrible ver todo eso vacío, cerrado.

EJ: Eso fue un suicidio, mejor dicho cuando se llevaron a Juan David ahí si ya no teníamos nada que hacer.

\section{La liquidación (2006 en adelante)}

EJ: Nosotros no sabíamos qué iba a pasar, pero hacíamos fila, era en la dirección del hospital y había policía adentro con ellos y más personas, y uno veía al policía, era la única cara amable que yo recuerdo, las demás eran de pánico porque la liquidadora nos hacía sentar.. yo creo que lo que nosotros sentimos fue lo que sintieron los judíos cuando los llevaron a 
la cámara de gas. Ella nos dejaba esperando y luego que a firmar ¿Cómo así que usted firme y no tiene derecho a nada?, ¿cómo que no tenemos derecho a nada? entonces la gente se ponía brava, se desordenaba y terminaba la reunión, entonces ella decía que temía por su vida y hasta perros llevaba. Yo firmé al final el 26 de diciembre porque sentí que me iba a enloquecer... ya no resistía más, físicamente y emocionalmente yo tenía como unas toneladas encima, ni dormía, todo el tiempo lloraba... me parecía que nada servía, y que todo era perder, entonces... caminar por el Materno era una tristeza.

AE: Unos lloraban, otros gritaban, otros firmaban, otros se iban, eso era un desastre. Pero a mí me llamaron para que fuera a firmar, pero yo dije, 'yo no voy a firmar porque es que yo sé que todos los derechos que tenemos ahí es algo que es de nosotros y no lo podemos entregar así no más'. Yo fui partícipe de no dejar firmar a muchas compañeras: yo les decía 'no firmen, no firmen que la convención es vigente, no firmen así duremos años peleando'. Yo pedí una ficha para entrar con Ana Karenina y entré, y me dijo '¿vas a firmar?', le dije 'no, yo vengo a hablar con usted', y me dijo 'pero primero firme y luego si', y dije 'no, es que uno primero habla y uno mira lo que va a comprar, es que uno no puede comprar algo que no es', entonces se quedó mirando y me dijo '¿qué es lo que quiere?' y le dije 'pues saber qué es lo que va a hacer con mis veinte años de trabajo acá porque es que yo no me puedo ir así, porque yo ya tengo tiempo para pensionarme, yo no me puedo ir así no más', y dijo 'pero es que ustedes están jóvenes para que trabajen', 'pero es que no importa, yo ya me gané mi pensión y usted no me la puede quitar así no más'. Le quité mi cédula y le dije 'yo no voy a firmar', entonces me dijo 'yo la anoto aquí en las especiales, las que nos dejaron de últimas para liquidar'. Ella liquidó lo que quiso, por lo menos a mi me salieron 20 millones, pero no es eso lo que realmente es.

TR: Lo más duro fue cuando entraron los negros esos (el ESMAD)... y yo no me acordé de mi corazón. Y una tomba cogió y manoseó a una compañera, ella entró llorando... yo estaba asustada, aterrorizada.

EJ: El subdirector del HLV con los pies le daba patadas a la puerta, yo lo veo todavía y eso no se me va a borrar, porque fue muy doloroso, sobre todo porque fue egresado de nosotros de la Nacional y del Materno... yo digo ¿porqué esa imagen no se me borra, o no quiero borrarla para que no se me olvide?

AE: Entonces entró La Victoria y eso eran celadores para acá y para allá, la gente venía menos porque ya había muchos inconvenientes, fuimos quedando pocos, algunos como que trataron de venir un tiempo... se fueron reduciendo hasta que no quedó ninguno... y yo tomé la decisión de venirme a vivir para acá con otra compañera. Yo venía todo el día y me quedaba la noche, mis hijos sabían que yo trabajaba acá, y yo vendía maní, galletas, ropa, y todas las artesanías, y duré como un año larguito eso fue del 2006 en adelante.

S: La permanencia en "La Carpa" se ha dificultado por la crisis laboral de los últimos dos años en que todo se ha puesto más duro: "a veces no tenemos ni para los buses".

TR: ¿Ahora a dónde llevaran esos chinitos, esos que recogen en el caño, en la calle? La salud se acabó, los médicos son mediocres... no confío en los médicos de ahora.

AE: En este momento yo no quisiera volver a trabajar en enfermería, ya no es mi fuerte. A mí me gustaba mucho y yo considero que fui una buena auxiliar, ahora, es una esclavitud muy grande, yo de ver que todo cambió acá, con las compañeras, el nuevo director las grita, las manda, las estruja, no se... yo creo que no me voy a poner en ese plan ni aquí ni en ningún otro lado, yo no trabajo en salud más. 
TR: Yo no sabía lo que era la depresión y ahora sí, con esto sí. Ya estoy cansada, aburrida no, cansada. Pierdo el hilo de las cosas, la concentración. La situación del IMI lo ha empeorado. Me preocupa mi vejez y mi plata. Trato de no pensar en nada de esto, trato de olvidar. Yo no aguanto más... uno va hasta donde puede, pero aguantar hambre no es posible.

TO: Uno ve que la realidad es otra... yo siempre he sentido que lo que perdimos fue muchísimo, ninguna plata nos va a recompensar, porque nadie nos va a garantizar una pensión buena... de todas maneras voy a seguir dependiendo económicamente yo sé que no voy a poder sola...

EJ: Una auxiliar de enfermería que uno la había visto que era hasta como de izquierda y de pronto resultaba que dios había enviado a la doctora Ana Karenina, pues uno dice que perdió la razón. Otra decía que niños que no han nacido ahí están rondado el Materno, a mi me da un poquito de miedo, porque ¿cómo dice sumercé que los que no han nacido están por ahí caminando porque no han podido nacer ahí?, pues uno dice ellas están como al borde...

S: Cuando me diagnosticaron el cáncer yo le pasé una carta a Ana Karenina diciéndole que me pagara el seguro. Cuando entró La Victoria me dijo, 'pues sisbenísese o pague como independiente. A mí nadie me obliga'.

EJ: No quiero dormir, algo pasa, le lucho al sueño.

TR: Con esta situación en cualquier momento yo vuelvo a infartar.

EJ: [Mi familia] estaba todo el tiempo pendiente, fue una depresión terrible. Bueno yo creo que la compañera que murió, ella tenía problemas depresivos, tenía tratamiento y manejó su problema, pero al final murió...

TR: La de azulito entró en estado de depresión, sin servicio médico y ella se encerró, se encerró, enloqueció y se murió.

S: Se han muerto 6 compañeras/os desde el 2007.

AE: Quién sabe hasta cuándo estaremos, ¿hasta cuándo será? Yo no puedo dejar de venir, vengo sábado, domingo, festivos y entre semana, cuando trabajaba era medio día o era en la noche cada tercer noche, pero ahora son todos los días, yo llego acá a las ocho, ocho y media y me estoy yendo a las cinco... es mucho tiempo.

EJ: ¿Por qué es que todos queremos volver al Materno? Porque en el Materno huele a líquido amniótico y a leche materna y uno quiere volver al útero, y uno quiere ir a la mamita y creo que todos queremos volver allá.

\section{DISCUSIÓN}

La acumulación flexible del neoliberalismo precisa la transformación de las subjetividades laborales para la sobreexplotación de las capacidades del trabajador (2-4). Estos atributos junto con la gestión de las empresas capitalistas de bienes y servicios se han transferido como control de gastos en salud a los hospitales en Colombia, que desde la Ley 100 se han transformado en empresas lucrativas, en las cuales los trabajadores 
viven la tensión entre facturar e imponer límites a los servicios prestados (13-16,24). Sabemos que las políticas neoliberales se han implementado violentamente, incluyendo asesinatos y debilitamientos al movimiento sindical $(3,25)$.

El caso del IMI refleja las formas como un Estado periférico resuelve la existencia de trabajadores/as de la salud con contratos públicos y con garantías laborales en un mercado transnacional privado. Múltiples violencias se narran y sienten en las voces y los cuerpos de estos/as trabajadoras. Entendemos que varias propuestas de interpretación de la violencia (política, estructural, simbólica y cotidiana) (26), son evidentes en el proceso de privatización del IMI, actuando por largos periodos de manera conexa pero con diferentes manifestaciones. Estas violencias constantes son de "baja intensidad" pero han aparecido con mucha fuerza como "tecnologías de violencia", definidas por Kleinman (27) para el caso del trauma político como aquellas tecnologías que pretenden tiranizar utilizando sensibilidades culturales y formas de interacción social que mantienen ocultas las críticas y las resistencias. En resumen Kleinman plantea que el trauma se usa sistemáticamente para silenciar a las personas a través de la generación de sufrimiento. (Foucault tiene un enfoque similar al hablar sobre tortura. Para el caso del IMI, estas tecnologías de violencia son evidentes, por ejemplo cuando trabajadores/as son presionadas para que renuncien o cuando entró La Victoria de la mano del ESMAD, causando no solo rupturas en la estructura física del IMI sino también resquebrajando su integridad emocional y mental.

Proponemos analizar las conexiones entre los diferentes tipos de violencia en el neoliberalismo y el dolor/sufrimiento de las mujeres bajo la categoría tortura ya que la unidad mente-cuerpo es justamente aquella que sustenta el ideario de acción de la tortura: al quebrar el cuerpo se quiebra la mente, y viceversa. La tortura se ha argumentado como componente necesario de los Estados Modernos, pero definirla no es sencillo, dadas las distinciones legales, morales y sentimentales de su uso (28). Para proponer avances en estas reflexiones y con fines de la presente investigación proponemos la siguiente definición de tortura: acciones violentas que causan dolor físicoemocional, ejecutadas por actores de poder sobre otros que desafían alterarlo y que están enmarcadas en principios morales, legales y emocionales de un ideario sobre el deber ser del futuro de la sociedad. Estos principios que sustentan lo que se considera el bien social mayor amenazado seguirán 
desarrollos históricos particulares $\mathrm{y}$, como consecuencia, las acciones de tortura se manifestarán de formas culturalmente diferenciadas. Para este caso, el bien social amenazado es la consolidación del modelo neoliberal de la salud en Colombia, en el cual, la acumulación flexible del capital mediante la venta de servicios en salud requiere del afianzamiento de gerencias mercantiles en las instituciones de salud y la transformación de las subjetividades de trabajadoras/es y sus formas de contratación.

El análisis sobre la díada torturador/torturado nos lleva a encontrar que efectivamente varios sujetos que torturan están presentes o actúan en distintos niveles, momentos y contextos, desde los más próximos como el ESMAD, hasta los más distantes (temporal y espacialmente), como quienes gestaron las políticas que han llevado a eliminar el IMI (las leyes 50 y 100), pasando por múltiples "torturadores" intermedios o intermediarios. El proceso de privatización del IMI como tortura nos obliga a preguntarnos también por los fines de la tortura en el contexto neoliberal de los trabajadores de la salud y discutir qué tanto se ajustan o no los eventos violentos del IMI con las características descritas como razones para torturar, como la eliminación de focos sociales considerados "contaminantes", el delatar cómplices de lo que se considera atenta contra el bien social mayor, o el encausamiento de conductas desviadas o potencialmente desviadas.

Hannah Arendt plantea que la tortura, la represión y el genocidio aparecen cuando la vida burocrática de las sociedades modernas ejerce una presión desmesurada sobre la esfera vital pública, entonces los burócratas no actúan siguiendo los principios de la ley, sino reglas administrativas y cuotas -lo que ella llama racionalidad instrumental $(28,29)$, es decir, la tensión entre democracia y burocracia de los Estados Modernos. Para el caso del IMI, señalamos a la agente liquidadora, Ana Karenina, como uno de los sujetos indispensables para su transformación, cumpliendo con los parámetros del sistema político moderno: "[e]n la edad moderna, la violencia no es ejercida necesariamente por el diablo, el tirano cruel, como ocurría en el pasado. En cambio, la ejerce el burócrata aburrido pero responsable" (28). Como limitación del enfoque de Arendt, llamado humanista, Rejali plantea que generalmente se denuncia el terror pero no se explica. Adicionalmente argumenta que la distinción clave entre el ámbito público y el de la administración oculta una asimetría importante, ya que por definición todo lo que no es público es considerado "administración", una apuesta hipotética de inversión del estado legal. Rejali señala que la 
tortura es obviamente administrada por las burocracias pero que es ejercida de formas muy distintas, lo cual imposibilita el análisis diferencial ya que todos los torturadores están clasificados como burocracia con una racionalidad eminentemente marcada en el postulado que tratan a los sujetos como objetos.

El Terrorismo de Estado, es planteado como una forma de gobernancia caracterizada por "procedimientos operativos estandarizados en múltiples centros de detención, aplicables a cientos de detenidos y usado con la aprobación e intención de las máximas autoridades" (Herman, citado en 28). Grandes complejos de tortura tienen relación con gobiernos extranjeros y con redes del capitalismo global y el interés de distintas empresas multinacionales (28).

Es evidente el funcionamiento del IMI en los últimos años como complejo de tortura, por lo menos en cuanto al confinamiento a un espacio, los interrogatorios, los abusos de poder, la alteración del Estado mental y las violaciones a los derechos humanos y laborales, sin dejar de señalar, además, las conexiones con los mercados globales en salud que necesitan que instituciones como el IMI desaparezcan para maximizar sus ganancias en Colombia. Como diferencia, sin embargo, queremos señalar que mientras en los complejos de tortura tradicionales la máxima es la destrucción del ser humano sin llevarlo a la muerte, en el caso del IMI, la máxima podría entenderse como la eliminación del sujeto, expresada en la muerte física (refiriéndonos a las 6 muertes) y en la muerte laboral, bien sea al llevarlo al abandono de la institución para dar paso al "progreso neoliberal en salud", o para que se conviertan en trabajadoras flexibles (más rentables para las nuevas empresas privadas en salud), que bien sea en su condición de desempleadas, jubiladas sin pensión o trabajadoras flexibles, satisfacen el deseo neoliberal de reducirle al Estado y a la empresa privada gastos de seguridad social.

Como conclusión planteamos que el avance del neoliberalismo en Colombia a la luz de las trabajadoras del IMI se hace evidente en múltiples violencias coexistentes que emergen en momentos diferentes como tortura y que se hacen visibles y palpables de diferentes maneras, obligando a trabajadoras/es a reconfigurar sus vidas y sus significados 
Agradecimientos: Agradecemos principalmente al grupo de trabajadoras/es del IMI, quienes nos han permitido acompañarles por estos años. Agradecemos al grupo de investigación Antropología Médica Crítica y a los asistentes a una presentación en el Seminario del Centro de Estudios Sociales por sus comentarios. Este proyecto fue financiado parcialmente por una beca de la CLACSO (Concurso: Cultura, poder y contra hegemonía. Año 2008. Categoría Semi senior) y por dos becas de la División de Investigación de la Universidad Nacional de Colombia (20101007624 año 2006-2008 y 201010013524 año 2009-2010).

\section{REFERENCIAS}

1. Antunes R [Internet]. El caracol y su concha: Ensayo sobre la nueva morfología del trabajo. Herramienta. 2006;31(Marzo). Disponible en: http://www.insumisos.com/bibliotecanew/ Nueva\%20morfologia\%20del\%20trabajo.pdf. Consultado agosto de 2009.

2. Antunes R. ¿Adiós al trabajo? Ensayo sobre las metamorfosis y el rol central del mundo del trabajo. 2 ed. Buenos Aires: Herramienta; 2003 [trad. 1995].

3. Antunes R. Los Sentidos del Trabajo: Ensayo sobre la afirmación y la negación del trabajo. Buenos Aires: Herramienta ediciones, Taller de Estudios Laborales; 2005.

4. García C. Las tecnologías empresariales del yo: La construcción de sujetos laborales en el contexto del trabajo inmaterial. Universitas Psychologica; 20076(1):49-58.

5. Giraldo C. ¿Protección o desprotección social? Bogotá: Ediciones Desde Abajo; 2007.

6. Almeida C. Reforma de sistemas de servicios de salud y equidad en América Latina y el Caribe: algunas lecciones de los años 80 y 90 . Cadernos de Saúde Publica 2002;18(4):905-925.

7. Kim JY, Millen JV, Irwin A, Gershman J (Ed). Dying for Growth. Global Inequality and the Health of the Poor. Monroe (Maine): Common Courage Press; 2000.

8. Tavares LRS. Ajuste Neoliberal e Desajuste Social na America Latina. Rio de Janeiro: UFRJ; 1999.

9. Escobar A. Encountering Development. The Making and Unmaking of the Third World. Princeton: Princeton University Press; 1995.

10. Iriart C, Merhy EE, Waitzkin H. Managed Care in Latin America: the new common sense in health policy reform. Social Science \& Medicine 2001;52:1243-1253.

11. Stocker K, Waitzkin H, Iriart C. The Exportation of Managed Care to Latin America. New England Journal of Medicine 1999;340(14):1131-1136.

12.CID. Evaluación de la Reforma Laboral (Ley 789 De 2002). Bogotá: CID, Universidad Nacional de Colombia; 2007.

13. García C. Subjetividades laborales: impacto de la reforma de la salud en trabajadores de tres hospitales públicos de Bogotá. Pre-Til. 2008;6(17):28-44.

14. García C. El hospital como empresa: nuevas prácticas, nuevos trabajadores. Universitas Psychologica. 2007;6(1):143-154.

15. Valdés E. La transformación de la gestión hospitalaria en el Distrito Capital. Pre-Til. 2008;6(17):64-84.

16. Defensoría del Pueblo. Autonomía médica y su relación con la prestación de los servicios. Bogotá: Defensoría del Pueblo; 2007.

17. Abadía-Barrero CE, Oviedo Manrique DG. Bureaucratic Itineraries in Colombia. A Theoretical and Methodological Tool to Assess Managed-Care Health Care Systems. Social Science \& Medicine. 2009;68(6):1153-1160.

18. Martínez A. [¿Calidad de la Atención o Atención con Calidad? Condiciones Laborales y Profesionales de la Salud en el Sistema General de Seguridad Social en Salud] 
Tesis de grado de Maestría en Salud Pública [2008]. Se localiza en: Universidad Nacional de Colombia, Bogotá.

19. Abadía-Barrero C, Navarrete HG, Pinilla Alfonso MY, Martínez Parra AG. En Alto Riesgo: La Crisis de la Salud Pública en el Instituto Materno Infantil de Bogotá. Antípoda. 2006; Julio-Diciembre(3):199-226.

20. Kleinman A, Das V, Lock M, editors. Social Suffering. Berkeley, Los Angeles, London: University of California Press; 1997.

21. Le Breton. Antropología del Dolor. Barcelona: Seix Barral; 1999.

22. Rogers AG, Casey ME, Ekert J, Holland J, Nakkula V, Sheinberg N. An Interpretive Poetics of Languages of the Unsayable. In: Josselson R, Lieblich A (Eds). Making Meaning of Narratives -in- The Narrative Study of Lives. Thousand Oaks, London, New Delhi: Sage Publications; 1999. p. 77-106.

23. Brugman J, Fierro M. Efectos de los aspectos diferenciales de exposición a la violencia relacionada con el desplazamiento forzado en el PTSD: mujeres desplazadas de la LMD en Colombia. Bogotá: Universidad de los Andes; 2009.

24. Abadía-Barrero C, Cortés G, Fino E, García C, Oviedo D, Pinilla Alfonso MY, et al. Perspectivas Inter-Situadas sobre el Capitalismo en la Salud: desde Colombia y sobre Colombia. Palimpsestus. 2007/2008;6:163-176.

25. Klein N. The Shock Doctrine. The Rise of Disaster Capitalism. New York: Metropolitan Books; 2007.

26. Bourgois P. The Continuum of Violence in War and Peace: Post-Cold War Lessons from El Salvador. En: Scheper-Hughes N, Bourgois P (Eds). Violence in War and Peace. Malden, Oxford, Victoria: Blackwell Publishing; 2004. p. 425-434.

27. Kleinman A. Writing at the Margin. Discourses Between Anthropology and Medicine. Berkeley, Los Angeles, London: University of California Press; 1995.

28. Rejali DM. Torture \& Modernity. Self, Society, and State in Modern Iran. Boulder, San Francisco, Oxford: Westview Press; 1994. 